Article

\title{
The Thermal Entropy Density of Spacetime
}

\section{Rongjia Yang}

${ }^{1}$ College of Physical Science and Technology, Hebei University, Baoding 071002, China;

E-Mail: yangrongjia@ @tsinghua.org.cn; Tel.: 86-158-3156-8995; Fax: 86-312-5079352.

${ }^{2}$ Department of Physics, Tsinghua University, Beijing 100084, China

Received: 28 November 2012 / Accepted: 25 December 2012 /

Published: 8 January 2013

\begin{abstract}
Introducing the notion of thermal entropy density via the first law of thermodynamics and assuming the Einstein equation as an equation of thermal state, we obtain the thermal entropy density of any arbitrary spacetime without assuming a temperature or a horizon. The results confirm that there is a profound connection between gravity and thermodynamics.
\end{abstract}

Keywords: thermal entropy density; gravity; thermodynamics

Classification: PACS 04.20.Cv, 05.70.Ce, 04.70.Dy

\section{Introduction}

In Newtonian theory of gravity, when a particle falls freely in a gravitational field, the gravity is also the inertial force. This fact leads to the principle that the inertial mass and the gravitational mass are equivalent. This principle can be considered as the first principle of equivalence. Based on this principle, Einstein suggested that the gravitational force and inertial force are equivalent. This equivalence between gravitational force and inertial force can be considered as the second principle of equivalence on which general relativity bases.

Besides these two relations (i.e., the relation between gravitational mass and inertial mass, and the relation between gravitational force and inertial force), it was also known that there is a profound connection between gravity and thermodynamics, as implied by the work of Cocke [1], Bekenstein [2], Hawking [3], Davies [4], and Unruh [5]. After these studies, Wald had shown that the entropy $S$ can be taken to be the Noether charge associated with the diffeomorphism invariance of the theory $[6,7]$ 
Jacobson revealed that the Einstein equation can be derived from the first law of thermodynamics [8]. This attempt had been generalized to the modified gravity [9-11] and had been revisited in [12], which was based on a consideration of the properties of a very small, spacelike two-plane in a uniformly accelerating motion. It had been shown that the field equations in both general relativity and Lovelock theories can be expressed as a thermodynamic identity near the horizon in a wide class of spacetime [13-15] (see a review [16]). Recently, the connection between gravity and thermodynamics had been proved to be held in the dynamical spacetime [17]. By using the maximum entropy principle to a charged perfect fluid, the generalized TolmanłOppenheimer-Volkoff is derived, which provides a strong evidence for the fundamental relationship between general relativity and ordinary thermodynamics [18]. In cosmological context, the Friedmann equation can be rewritten in the form of the first law of thermodynamics [19-28]. In [29], it has been explicitly shown that the equations of motion for modified gravity theories of $F(R)$-gravity, the scalar-Gauss-Bonnet gravity, $F(\mathcal{G})$-gravity and the non-local gravity are equivalent to the Clausius relation in thermodynamics. In [30], Verlinde argued that gravity can be explained as entropic force. Recently, it was shown that the Einstein-Hilbert action can be constructed by minimizing free energy [31].

All these investigations were carried out in special contexts, or based on some assumptions, such as Unruh temperature, the existence of horizon, null surfaces, the apparent horizon, and so on. The key point in Jacobson's analysis, for example, is based on three assumptions: the first law of thermodynamics holds, the temperature experienced by the observer is the Unruh temperature, and the heat flow through the past Rindler horizon is defined to be the boost-energy current carried by matter. Does the connection between gravity and thermodynamics hold in any arbitrary spacetime? Can the analysis be carried out without assuming a specific expression of temperature or horizon? The difficulties are that we cannot find a general expression of temperature (to find a specific expression of temperature, such as Unruh temperature taken by Jacobson or Hawking temperature taken by other researchers on horizon) or define a horizon in any arbitrary spacetime. In this paper, we try to investigate the relation between gravity and thermodynamics without firstly assuming a temperature or a horizon. The result we obtain implies that gravity possesses thermal effects, or, thermal entropy density possesses effects of gravity.

\section{Thermal Entropy Density of Spacetime}

In general relativity or thermodynamics, both the energy density $\rho$ and the pressure $p$ play important roles. In general relativity, the energy density and the pressure are contained in the stress-energy tensor. In thermodynamics, $\rho$ and $p$ are contained in the first law of thermodynamics. We can reasonably conjecture that a relation may exist between gravitation and thermodynamics. So let us begin with the first law of thermodynamics in curved spacetime

$$
d E=T d S-p d V
$$

where $E$ is the total energy and $S$ is entropy within the volume $V, T$ is the temperature and $p$ is the pressure of the perfect fluid, and $d V=\sqrt{h} d^{3} x$ with $\sqrt{h}$ being the determinant of the spatial metric. Throughout this paper, we take $c=G=1$ and use metric signature $(-,+,+,+)$. For a very small volume, the energy density can be considered as unchanged, so Equation (1) can be rewritten as

$$
(\rho+p) d V=T d S \equiv T s d V
$$


where $s$ is the entropy density. To avoid the difficulty of finding a specific expression of temperature, we introduce the thermal entropy density defined as $\sigma \equiv T s$, and re-express Equation (2) as

$$
\sigma=\rho+p
$$

For radiation, $\rho=\alpha T^{4}$ with $\alpha$ being a constant, $p=\rho / 3$, and $s=4 \alpha T^{3} / 3$. It is obvious that we have $\sigma \equiv T s=\rho+p$. If $p=w \rho$ with $w$ being a constant, the thermal entropy density is proportional to the energy density, and the change of the thermal entropy density with time is also proportional to that of the energy density, $\dot{\sigma} \propto \dot{\rho}$, for $w>0$. For dust (or dark matter) with $p \simeq 0$, the thermal entropy density is just the energy density. For $w<0$, the thermal entropy increases when the energy density decreases, and vice versa. The energy density $\rho$ and the pressure $p$ can be observed, so does the thermal entropy density. So it can be concluded that the thermal entropy density is a notion that is more comprehensive than the notion of the energy density in thermodynamics.

In order to relate the thermal entropy density with general relativity, we briefly review the main point in [8]. Assuming that the first law of thermodynamics holds, namely $\delta Q=T d S$, with $\delta Q$ and $T$ interpreted as the energy flux and Unruh temperature, Jacobson obtained the Einstein equation. In other words, viewed in thermodynamics way, the Einstein equation can be thought of as a thermal state. Here we assume that both the first law of thermodynamics and the Einstein equation hold, and adopt the view obtained in [8]: the Einstein equation is an equation of state. So we can treat thermodynamic quantity $\rho+p$ as a bridge to relate the thermal entropy density and the geometrical quantities of spacetime. Let us look for the expression of $\rho+p$ in general relativity so as to find the relation between gravity and thermodynamics. Thanks to the Einstein equation [32]

$$
R_{\mu \nu}-\frac{1}{2} g_{\mu \nu} R+\Lambda g_{\mu \nu}=8 \pi T_{\mu \nu}
$$

and the stress energy tensor of the perfect fluid

$$
T_{\mu \nu}=g_{\mu \nu} p+(\rho+p) u_{\mu} u_{\nu}
$$

we obtain

$$
R-4 \Lambda=-8 \pi(3 p-\rho)
$$

To obtain the expression of $\rho+p$ in general relativity, we must find another equation about $\rho$ or $p$. We use the $3+1$ Einstein equation to attain this goal. Let $n^{\mu}$ be the unit normal vector field to the three-dimensional hypersurfaces $\Sigma$, then we have [32,33]

$$
n^{\mu} n^{\nu} R_{\mu \nu}+\frac{1}{2} R-\Lambda=8 \pi \mathcal{E}
$$

where $\mathcal{E}=\Gamma^{2}(\rho+p)-p$ with $\Gamma$ being the Lorentz factor. According to the scalar Gauss relation, we obtain

$$
\mathcal{R}+K^{2}-K_{i j} K^{i j}-2 \Lambda=16 \pi \mathcal{E}
$$


where $\mathcal{R}$ is Ricci scalar of the three-dimensional hypersurfaces $\Sigma, K_{i j}$ is the extrinsic curvature tensor of $\Sigma$, and $K$ is the trace of the $K_{i j}$. Combining Equations (6) and (8), we obtain the expression of $\rho+p$ in general relativity

$$
\rho+p=\frac{1}{4 \pi\left(4 \Gamma^{2}-1\right)}\left[\mathcal{R}+K^{2}-K_{i j} K^{i j}-\frac{1}{2} R\right]
$$

From Equations (3) and (9), one can easily get

$$
\sigma=\frac{1}{4 \pi\left(4 \Gamma^{2}-1\right)}\left[\mathcal{R}+K^{2}-K_{i j} K^{i j}-\frac{1}{2} R\right]
$$

The four-dimensional Ricci scalar, $R$, can be decomposed as [33]

$$
R=\mathcal{R}+K^{2}+K_{i j} K^{i j}-\frac{2}{N} \mathcal{L}_{m} K-\frac{2}{N} D_{i} D^{i} N
$$

where $\mathcal{L}_{m}$ is the Lie derivative along $\mathbf{m}$ of any vector tangent to $\Sigma$, and $D_{i}$ is the Levi-Civita connection associated with the metric of the three-dimensional hypersurfaces $\Sigma$. Then we can express the thermal entropy density with three-dimensional spatial geometrical quantities as

$$
\sigma=\frac{1}{8 \pi\left(4 \Gamma^{2}-1\right)} \times\left[\mathcal{R}+K^{2}-3 K_{i j} K^{i j}+\frac{2}{N} \mathcal{L}_{m} K+\frac{2}{N} D_{i} D^{i} N\right]
$$

Equations (10) and (12) are the most important result we obtained in this work. The left-hand side of the equation is a quantity concerned with thermodynamics, while the right-hand side of the equation is related to the geometrical quantities of the spacetime. According to Equations (10) and (12), they are equivalent. Recall the case in Newtonian theory of gravity, when a particle free falls in a gravitational field, the gravity is also the inertial force, one can see that the inertial mass and the gravitational mass are equivalent. Now, the energy density $\rho$ and the pressure $p$ are not only the source of heat but also the source of gravity. This fact leads to Equations (10) and (12), which imply that gravity possesses thermal effects, or, thermal entropy density possesses effects of gravity. We note that Equations (10) and (12) hold for perfect fluid only, and the case for non-perfect fluid will be discussed elsewhere.

In co-moving coordinate, Equation (10) takes the form [34]

$$
\sigma=\frac{1}{24 \pi}\left(4 R_{\mathrm{s}}-3 R\right)
$$

where $R_{\mathrm{s}}=g_{11} R^{11}+g_{22} R^{22}+g_{33} R^{33}$. In FRW universe, the thermal entropy density of the spacetime is $-\left(\dot{H}-k / a^{2}\right) / 4 \pi$ with $H=\dot{a} / a$ being the Hubble parameter and $k$ being a constant. In radiation dominated era, according to the Einstein equation, we have $-\left(\dot{H}-k / a^{2}\right) / 4 \pi=\rho+p=4 \alpha T^{4} / 3=$ $T s \equiv \sigma$ with $\alpha=8 \pi^{5} k_{\mathrm{B}}^{4} /\left(15 h^{3}\right)$ and $s=4 \alpha T^{3} / 3$ [35], so Equation (12) holds.

\section{Conclusions}

We have introduced the notion of thermal entropy density via the first law of thermodynamics, and related it with three dimensional spatial geometrical quantities via the Einstein equation. We have obtained the thermal entropy density of any arbitrary spacetime without assuming a temperature or a horizon, that is to say, gravity can possess thermal effects, or, thermal entropy density can possess 
effects of gravity. The results also indicate that, besides gravity, the thermal entropy density can also be geometrized. The results we obtained confirm that there is a profound connection between gravity and thermodynamics. The thermal entropy density of spacetime can be applied to discuss the gravitational collapse. Here we have discussed the case of perfect fluid only and leave the case of non-perfect fluid for future investigations.

\section{Acknowledgments}

This study is supported in part by National Natural Science Foundation of China under Grant Nos. 11147028 and 11273010, Hebei Provincial Natural Science Foundation of China under Grant No. A2011201147, and Research Fund for Doctoral Programs of Hebei University under Grant No. 2009-155.

\section{References}

1. Cocke, W.J. A maximum entropy principle in general relativity and the stability of fluid spheres. Ann. Inst. Henri Poincaré 1965, 2, 283.

2. Bekenstein, J.D. Black holes and entropy-Bekenstein. Phys. Rev. D 1973, 7, 2333-2346.

3. Hawking, S.W. Particle creation by black holes. Commun. Math. Phys. 1975, 43, 199-220.

4. Davies, P.C.W. Scalar particle production in Schwarzschild and Rindler metrics. J. Phys. A 1975, 8, 609-616.

5. Unruh, W.G. Notes on black hole evaporation. Phys. Rev. D 1976, 14, 870.

6. Wald, R.M. Black hole entropy is the Noether charge. Phys. Rev. D 1993, 48, 3427-3431.

7. Iyer, V.; Wald, R.M. Some properties of Noether charge and a proposal for dynamical black hole entropy. Phys. Rev. D 1994, 50, 846-864.

8. Jacobson, T. Thermodynamics of sacetime: The einstein equation of state. Phys. Rev. Lett. 1995, $75,1260-1263$.

9. Eling, C.; Guedens, R.; Jacobson ,T. Non-equilibrium thermodynamics of spacetime. Phys. Rev. Lett. 2006, 96, 121301.

10. Elizalde, E.; Silva, P.J. $F(R)$ gravity equation of state. Phys. Rev. D 2008, 78, 061501.

11. Brustein, R.; Hadad, M. The Einstein equations for generalized theories of gravity and the thermodynamic relation delta $Q=T \delta S$ are equivalent. Phys. Rev. Lett. 2009, 103, 101301.

12. Makela, J.; Peltola, A. Gravitation and thermodynamics: The Einstein equation of state revisited. Int. J. Mod. Phys. D 2009, 18, 669-689.

13. Padmanabhan, T. Classical and quantum thermodynamics of horizons in spherically symmetric space-times. Class. Quan. Grav. 2002, 19, 5387-5408.

14. Paranjape, A.; Sarkar, S.; Padmanabhan, T. Thermodynamic route to field equations in Lancos-Lovelock gravity. Phys. Rev. D 2006, 74, 104015.

15. Kothawala, D.; Sarkar, S.; Padmanabhan, T. Einstein's equations as a thermodynamic identity: The Cases of stationary axisymmetric horizons and evolving spherically symmetric horizons. Phys. Lett. B 2007, 652, 338-342. 
16. Padmanabhan, T. Thermodynamical aspects of gravity: New insights. Rep. Prog. Phys. 2010, 73, 046901.

17. Wu, S.F.; Wang, B.; Ge, X.H.; Yang, G.H. Gravitational thermodynamics and universal holographic duality in dynamical spacetimes. arXiv:1109.0193.

18. Gao, S. A general maximum entropy principle for self-gravitating perfect fluid. Phys. Rev. D 2011, 84, 104023.

19. Danielsson, U.H. Transplanckian energy production and slow roll inflation. Phys. Rev. D 2005, $71,023516$.

20. Frolov, A.V.; Kofman, L. Inflation and de Sitter thermodynamics. J. Cosmol. Astropart. Phys. 2003, 05, 009.

21. Calcagni, G. de Sitter thermodynamics and the braneworld. J. High Energy Phys. 2005, 0509, 060.

22. Cai, R.G.; Kim, S.P. First law of thermodynamics and Friedmann equations of Friedmann-Robertson-Walker universe. J. High Energy Phys. 2005, 02, 050.

23. Cai, R.G.; Cao, L.M.; Ohta, N. Unified first law and thermodynamics of apparent horizon in FRW universe. Phys. Rev. D 2010, 81, 061501(R).

24. Akbar, M.; Cai, R.G. Thermodynamic behavior of friedmann equations at apparent horizon of FRW universe. Phys. Rev. D 2007, 75, 084003.

25. Gong, Y.; Wang, A. The Friedmann equations and thermodynamics of apparent horizons. Phys. Rev. Lett. 2007, 99, 211301.

26. Sheykhi, A.; Wang, B.; Cai ,R.G. Deep connection between thermodynamics and gravity in Gauss-Bonnet braneworld. Phys. Rev. D 2007, 76, 023515.

27. Wu, S.F.; Wang, B.; Yang, G.H. Thermodynamics on the apparent horizon in generalized gravity theories. Nucl. Phys. B 2008, 799, 330-344.

28. Yang, R.-J. Bound on the equation of state of dark energy from the Generalized second law of thermodynamics. Int. J. Theor. Phys. 2012, 51, 1692.

29. Bamba, K.; Geng, C.Q.; Nojiri, S.; Odintsov, S.D. Equivalence of modified gravity equation to the Clausius relation. Europhys. Lett. 2010, 89, 50003.

30. Verlinde, E.P. On the origin of gravity and the laws of newton. J. High Energy Phys. 2011, 4, 029.

31. Bracken, P. The Einstein-Hilbert Action Horizons and Connections with Thermodynamics. arXiv: 1111.5068

32. Wald, R.M. General Relativity; The University of Chicago Press: Chicago, USA, 1984.

33. Gourgoulhon, E. 3+1 Formalism and Bases of Numerical Relativity. arXiv:gr-qc/0703035

34. Yang, R.-J. Thermal entropy density in co-moving spacetime. Unpublished work.

35. Weinberg, S. Gravitation and Cosmology: Principles and Applications of the Greneral Theory of Relativity; John Wiley: Hoboken, NJ, USA, 1972.

(c) 2013 by the author; licensee MDPI, Basel, Switzerland. This article is an open access article distributed under the terms and conditions of the Creative Commons Attribution license (http://creativecommons.org/licenses/by/3.0/). 